\title{
Analysis of a Production System with a Feedback Buffer and a General Dispatching Time
}

\author{
HO WOO LEE* and BOO YONG AHN \\ Department of Industrial Engineering, Sung Kyun Kwan University, \\ Su Won, 440-746, Republic of Korea
}

(Received December 1998; In final form 13 May 1999)

In this paper, we analyze a production system with a finite feedback buffer and dispatching time. Parts enter a "main buffer" before they are processed. Processed parts leave the system with probability $1-p$ or are fed back to a "feedback buffer" with probability $p$. As soon as the feedback buffer becomes full, the parts in the feedback buffer are dispatched, all at once, to the main buffer by the server for reprocessing. The dispatching time follows a general distribution. Thus the server is engaged either in one of the following states: idle, processing, dispatching.

We derive various performance measures such as the mean number of parts in each buffer, the mean system sojourn time and the dispatching rate. We also discuss the effects of the dispatching time on the performance measures. We finally derive the procedure to obtain the optimal buffer size that minimizes the overall operating cost.

Keywords: $M / G / 1$ feedback queue; Dispatching; Production system

\section{INTRODUCTION}

We consider a production system in which processed parts may need to be fed back for reprocessing. Studies on feedback systems include Takács [8], Disney et al. [3], Disney [2], Foley and Disney [4], Pourbabai [6], D'avignon and Disney [1], Schrage [7], Takine et al. [9], and Wortman et al. [10], to name a few. But these studies assume that

\footnotetext{
*Corresponding author. E-mail: hwlee@yurim.skku.ac.kr.
} 
the feedback parts are returned to the waiting buffer instantaneously or after a random time. Recently, Lee and Seo [5] studied a feedback system in which fedback parts wait in an intermediate feedback buffer until they are dispatched to the main buffer. In their system, new arrivals enter the main buffer before processing. Defective items are fed back into an intermediate buffer. As soon as the feedback buffer is fully occupied, the parts in the feedback buffer are dispatched instantaneously to the main buffer, all at once, for reprocessing. This paper extends their model such that non-zero random dispatching time is incorporated into the model. As far as mathematical analysis is concerned, the non-zero dispatching time adds a dimension to the model and increases the complexity of the solution procedure.

We, first, develop the system equations, then, we derive the joint transform of the number of parts in the main buffer, the number of parts in the feedback buffer and the remaining times. Various performance measures such as the mean number of parts in each buffer, system sojourn time, dispatching rate, and idle/busy probabilities are obtained. Finally a procedure to compute the optimal size of the feedback buffer is presented.

\section{THE SYSTEM, MODEL AND SYSTEM EQUATIONS}

We consider a production system which operates as follows (Fig. 1):

(1) New parts arrive according to a Poisson process with rate $\lambda$.

(2) The system has two buffers: a main buffer and a feedback buffer.

(3) New parts enter the main buffer to be processed by a server.

(4) Processing times are iid general random variables.

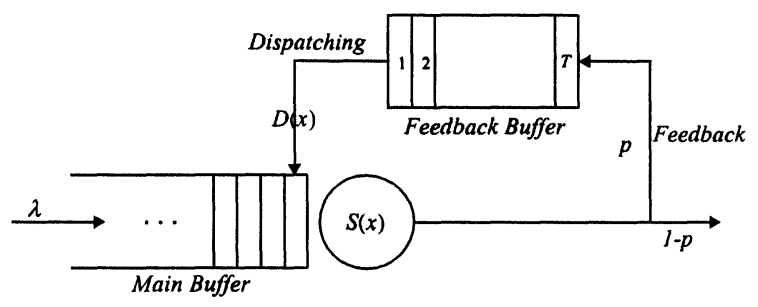

FIGURE 1 The system. 
(5) The system has the Bernoulli scheduling. That is, a processed part is instantaneously merged into the feedback buffer with probability $p$ or leaves the system with probability $1-p$.

(6) The capacity of the main buffer is $\infty$. The capacity of the feedback buffer is $T$.

(7) As soon as the feedback buffer becomes full, all parts are dispatched to the main buffer.

(8) The server pauses processing while engaged in dispatching. Thus, the server is either in a processing state, in a dispatching state, or in an idle state.

(9) The dispatching time, i.e., the time that it takes for the server to dispatch the parts from the feedback buffer to the main buffer is a continuous random variable that follows a general distribution. As soon as the dispatching job is finished, the server resumes processing the parts.

(10) The processing times and the dispatching times are independent.

Let us define the following notations:

\begin{tabular}{|c|c|}
\hline$N_{\mathbf{M}}(t)$ & $\begin{array}{l}\text { Number of parts in the main buffer at time } t \text {, } \\
\text { including the one being processed }\end{array}$ \\
\hline$N_{\mathrm{F}}(t)$ & $\begin{array}{l}\text { number of parts in the feedback buffer at } \\
\text { time } t\end{array}$ \\
\hline$N_{\mathrm{D}}(t)$ & number of parts being dispatched at time $t$ \\
\hline$T$ & $\begin{array}{l}\text { dispatching threshold (capacity of the } \\
\text { feedback buffer) }\end{array}$ \\
\hline$S$ & processing time, a random variable \\
\hline$s(x)$ & $\begin{array}{l}\text { probability density function (pdf) of the } \\
\text { processing time }\end{array}$ \\
\hline$S(x)$ & $\begin{array}{l}\text { distribution function (DF) of the } \\
\text { processing time }\end{array}$ \\
\hline$S_{\mathrm{R}}(t)$ & remaining processing time at $t$ \\
\hline$D$ & dispatching time, a random variable \\
\hline$d(x)$ & pdf of $D$ \\
\hline$D(x)$ & DF of $D$ \\
\hline$D_{\mathrm{R}}(t)$ & remaining dispatching time at $t$ \\
\hline$E(V)$ & mean of random variable $V$ \\
\hline$E\left(V^{2}\right)$ & second moment of $V$ \\
\hline
\end{tabular}


$V^{*}(\theta)$

$\operatorname{Var}(V)$

$L_{\mathbf{M}}=\lim _{t \rightarrow \infty} E\left[N_{\mathbf{M}}(t)\right]$

$L_{\mathrm{F}}=\lim _{t \rightarrow \infty} E\left[N_{\mathrm{F}}(t)\right]$
Laplace transform of the pdf of $V$ variance of $V$

steady-state mean number of parts in the main buffer (including the one in processing).

$L_{\mathrm{D}}=\lim _{t \rightarrow \infty} E\left[N_{\mathrm{D}}(t)\right]$ steady-state mean number of parts being dispatched

$L=L_{\mathrm{M}}+L_{\mathrm{F}}+L_{\mathrm{D}}$

$W$

$\alpha(T)$ mean sojourn time of a part in the system dispatching rate as a function of $T$

$Y(t)= \begin{cases}0, & \text { operator is idle } \\ 1, & \text { operator is processing } \\ 2, & \text { operator is dispatching }\end{cases}$

$U_{j}(t)=\operatorname{Pr}\left[N_{\mathrm{M}}(t)=0, N_{\mathrm{F}}(t)=j, Y(t)=0\right] \quad(0 \leq j \leq T-1)$

$U_{j}=\lim _{t \rightarrow \infty} U_{j}(t)$

$P_{i, j}(x, t)=\operatorname{Pr}\left[N_{\mathrm{M}}(t)=i, N_{\mathrm{F}}(t)=j, S_{\mathrm{R}}(t) \in(x, x+\mathrm{d} x), Y(t)=1\right]$

$(i \geq 1,0 \leq j \leq T-1)$

$P_{i j}(x)=\lim _{t \rightarrow \infty} P_{i j}(x, t)$

$Q_{i}(y, t)=\operatorname{Pr}\left[N_{\mathrm{M}}(t)=i, N_{\mathrm{D}}(t)=T, D_{\mathrm{R}}(t) \in(y, y+\mathrm{d} y), Y(t)=2\right]$

$(i \geq 0)$

$Q_{i}(y)=\lim _{t \rightarrow \infty} Q_{i}(y, t)$

\subsection{System Equations}

It is not difficult to derive the following steady-state system equations:

$$
\begin{gathered}
0=-\lambda U_{0}+(1-p) P_{1,0}(0), \\
0=-\lambda U_{j}+(1-p) P_{1, j}(0)+p P_{1, j-1}(0) \quad(1 \leq j \leq T-1), \\
-\frac{\mathrm{d}}{\mathrm{d} x} P_{1,0}(x)=-\lambda P_{1,0}(x)+U_{0} \lambda s(x)+(1-p) P_{2,0}(0) s(x), \\
-\frac{\mathrm{d}}{\mathrm{d} x} P_{i, 0}(x)=-\lambda P_{i, 0}(x)+P_{i-1,0}(x) \lambda+(1-p) P_{i+1,0}(0) s(x) \\
(2 \leq i \leq T-1),
\end{gathered}
$$




$$
\begin{gathered}
-\frac{\mathrm{d}}{\mathrm{d} x} P_{i, 0}(x)=-\lambda P_{i, 0}(x)+P_{i-1,0}(x) \lambda+(1-p) P_{i+1,0}(0) s(x) \\
+Q_{i-T}(0) s(x) \quad(i \geq T) \\
-\frac{\mathrm{d}}{\mathrm{d} x} P_{1, j}(x)=-\lambda P_{1, j}(x)+U_{j} \lambda s(x)+(1-p) P_{2, j}(0) s(x) \\
\quad+p P_{2, j-1}(0) s(x) \quad(1 \leq j \leq T-1) \\
-\frac{\mathrm{d}}{\mathrm{d} x} P_{i, j}(x)=-\lambda P_{i, j}(x)+P_{i-1, j}(x) \lambda \\
\quad+(1-p) P_{i+1, j}(0) s(x)+p P_{i+1, j-1}(0) s(x) \\
\quad(i \geq 2,1 \leq j \leq T-1) \\
-\frac{\mathrm{d}}{\mathrm{d} y} Q_{0}(y)=-\lambda Q_{0}(y)+p P_{1, T-1}(0) \mathrm{d}(y) \\
-\frac{\mathrm{d}}{\mathrm{d} y} Q_{i}(y)=-\lambda Q_{i}(y)+Q_{i-1}(y) \lambda+p P_{i+1, T-1}(0) \mathrm{d}(y)
\end{gathered}
$$

Let us define the Laplace transforms as follows:

$$
\begin{aligned}
P_{i, j}^{*}(\theta) & =\int_{0}^{\infty} \mathrm{e}^{-\theta x} P_{i, j}(x) \mathrm{d} x \quad(i \geq 1,0 \leq j \leq T-1), \\
Q_{i}^{*}(\theta) & =\int_{0}^{\infty} \mathrm{e}^{-\theta y} Q_{i}(y) \mathrm{d} y \quad(i \geq 0) .
\end{aligned}
$$

By taking the Laplace transform of the Eqs. (2.3)-(2.9), we get the following transform equations:

$$
\begin{aligned}
& (\lambda-\theta) P_{1,0}^{*}(\theta)=\lambda U_{0} S^{*}(\theta)+(1-p) P_{2,0}(0) S^{*}(\theta)-P_{1,0}(0), \\
& (\lambda-\theta) P_{i, 0}^{*}(\theta)=\lambda P_{i-1,0}^{*}(\theta)+(1-p) P_{i+1,0}(0) S^{*}(\theta)-P_{i, 0}(0) \\
& \quad(2 \leq i \leq T-1),
\end{aligned}
$$




$$
\begin{gathered}
(\lambda-\theta) P_{i, 0}^{*}(\theta)=\lambda P_{i-1,0}^{*}(\theta)+(1-p) P_{i+1,0}(0) S^{*}(\theta) \\
+Q_{i-T}(0) S^{*}(\theta)-P_{i, 0}(0) \quad(i \geq T), \quad(2.12) \\
(\lambda-\theta) P_{1, j}^{*}(\theta)=\lambda U_{j} S^{*}(\theta)+(1-p) P_{2, j}(0) S^{*}(\theta) \\
+p P_{2, j-1}(0) S^{*}(\theta)-P_{1, j}(0) \quad(1 \leq j \leq T-1) \\
(\lambda-\theta) P_{1, j}^{*}(\theta)=\lambda P_{i-1, j}^{*}(\theta)+(1-p) P_{i+1, j}(0) S^{*}(\theta) \\
+p P_{i+1, j-1}(0) S^{*}(\theta)-P_{i, j}(0) \quad(i \geq 2, \quad 1 \leq j \leq T-1) \\
(\lambda-\theta) Q_{0}^{*}(\theta)=p P_{1, T-1}(0) D^{*}(\theta)-Q_{0}(0), \\
(\lambda-\theta) Q_{i}^{*}(\theta)=\lambda Q_{i-1}^{*}(\theta)+p P_{i+1, T-1}(0) D^{*}(\theta)-Q_{i}(0) \quad(i \geq 1)
\end{gathered}
$$

\subsection{Analysis}

Let us define the following generating functions with respect to $z_{2}$, for $\left|z_{2}\right| \leq 1$ :

$$
\begin{gathered}
U\left(z_{2}\right)=\sum_{j=0}^{T-1} U_{j} z_{2}^{j}, \quad P_{i, \cdot}^{*}\left(\theta, z_{2}\right)=\sum_{j=0}^{T-1} P_{i, j}^{*}(\theta) z_{2}^{j}, \\
P_{i, \cdot}\left(0, z_{2}\right)=\sum_{j=0}^{T-1} P_{i, j}(0) z_{2}^{j} .
\end{gathered}
$$

From Eqs. (2.1) and (2.2), we get

$$
\begin{gathered}
\lambda U_{0}=(1-p) P_{1,0}(0), \\
\lambda \sum_{j=1}^{T-1} U_{j} z_{2}^{j}=(1-p) \sum_{j=1}^{T-1} P_{1, j}(0) z_{2}^{j}+p \sum_{j=1}^{T-1} P_{1, j-1}(0) z_{2}^{j},
\end{gathered}
$$


which are simplified to

$$
\lambda U\left(z_{2}\right)=\left(1-p+p z_{2}\right) P_{1, \cdot}\left(0, z_{2}\right)-p z_{2}^{T} P_{1, T-1}(0) .
$$

Similarly, from Eq. (2.10) and (2.13), we get

$$
\begin{aligned}
(\lambda-\theta) P_{1, \cdot}^{*}\left(\theta, z_{2}\right)= & \lambda S^{*}(\theta) U\left(z_{2}\right)+\left(1-p+p z_{2}\right) S^{*}(\theta) P_{2, \cdot}\left(0, z_{2}\right) \\
& -p S^{*}(\theta) z_{2}^{T} P_{2, T-1}(0)-P_{1, \cdot}\left(0, z_{2}\right)
\end{aligned}
$$

From Eqs. (2.11) and (2.14), we get

$$
\begin{aligned}
(\lambda-\theta) P_{1, \cdot}^{*}\left(\theta, z_{2}\right)= & \lambda P_{i-1, \cdot}^{*}\left(\theta, z_{2}\right)+\left(1-p+p z_{2}\right) S^{*}(\theta) P_{i+1, \cdot}\left(0, z_{2}\right) \\
- & p S^{*}(\theta) z_{2}^{T} P_{i+1, T-1}(0)-P_{i, \cdot}\left(0, z_{2}\right) \\
& (2 \leq i \leq T-1) .
\end{aligned}
$$

From Eqs. (2.12) and (2.14), we get

$$
\begin{aligned}
(\lambda-\theta) P_{1, \cdot}^{*}\left(\theta, z_{2}\right)= & \lambda P_{i-1, \cdot}^{*}\left(\theta, z_{2}\right)+\left(1-p+p z_{2}\right) S^{*}(\theta) P_{i+1, \cdot}\left(0, z_{2}\right) \\
& -p S^{*}(\theta) z_{2}^{T} P_{i+1, T-1}(0) \\
& +Q_{i-T}(0) S^{*}(\theta)-P_{i, \cdot}\left(0, z_{2}\right) \quad(i \geq T) .
\end{aligned}
$$

For $z_{1}$ on $\left|z_{1}\right| \leq 1$, we define the following generating functions:

$$
\begin{gathered}
P^{*}\left(\theta, z_{1}, z_{2}\right)=\sum_{i=1}^{\infty} P_{i, \cdot}^{*}\left(\theta, z_{2}\right) z_{1}^{i}, \quad P\left(0, z_{1}, z_{2}\right)=\sum_{i=1}^{\infty} P_{i, \cdot}\left(0, z_{2}\right) z_{1}^{i}, \\
P_{\cdot, T-1}\left(0, z_{1}\right)=\sum_{i=1}^{\infty} P_{i, T-1}(0) z_{1}^{i}, \quad Q^{*}\left(\theta, z_{1}\right)=\sum_{i=0}^{\infty} Q_{i}^{*}(\theta) z_{1}^{i} \\
Q\left(0, z_{1}\right)=\sum_{i=0}^{\infty} Q_{i}(0) z_{1}^{i} .
\end{gathered}
$$


From Eqs. (2.18)-(2.20), after using Eq. (2.17), we get

$$
\begin{aligned}
(\lambda- & \left.\lambda z_{1}-\theta\right) P^{*}\left(\theta, z_{1}, z_{2}\right) \\
= & \lambda\left(z_{1}-1\right) S^{*}(\theta) U\left(z_{2}\right)+\frac{\left(1-p+p z_{2}\right) S^{*}(\theta)-z_{1}}{z_{1}} P\left(0, z_{1}, z_{2}\right) \\
& \quad-\frac{p S^{*}(\theta) z_{2}^{T}}{z_{1}} P_{\cdot, T-1}\left(0, z_{1}\right)+S^{*}(\theta) Q\left(0, z_{1}\right) z_{1}^{T} .
\end{aligned}
$$

From Eqs. (2.15) and (2.16), we get

$$
\left(\lambda-\lambda z_{1}-\theta\right) Q^{*}\left(\theta, z_{1}\right)=\frac{p D^{*}(\theta)}{z_{1}} P_{,, T-1}\left(0, z_{1}\right)-Q\left(0, z_{1}\right) .
$$

Let $\theta=\lambda-\lambda z_{1}$ in Eq. (2.22) to eliminate $Q^{*}\left(\theta, z_{1}\right)$ and get

$$
Q\left(0, z_{1}\right)=\frac{p D^{*}\left(\lambda-\lambda z_{1}\right)}{z_{1}} P_{\cdot, T-1}\left(0, z_{1}\right)
$$

Use Eq. (2.23) in (2.21) and (2.22) to get

$$
\begin{aligned}
(\lambda- & \left.\lambda z_{1}-\theta\right) P^{*}\left(\theta, z_{1}, z_{2}\right) \\
= & \lambda\left(z_{1}-1\right) S^{*}(\theta) U\left(z_{2}\right)+\frac{\left(1-p+p z_{2}\right) S^{*}(\theta)-z_{1}}{z_{1}} P\left(0, z_{1}, z_{2}\right) \\
& \quad+\frac{p S^{*}(\theta)\left[z_{1}^{T} D^{*}\left(\lambda-\lambda z_{1}\right)-z_{2}^{T}\right]}{z_{1}} P_{\cdot, T-1}\left(0, z_{1}\right), \\
& \left(\lambda-\lambda z_{1}-\theta\right) Q^{*}\left(\theta, z_{1}\right)=\frac{p\left[D^{*}(\theta)-D^{*}\left(\lambda-\lambda z_{1}\right)\right]}{z_{1}} P_{\cdot, T-1}\left(0, z_{1}\right) .
\end{aligned}
$$

Let $\theta=\lambda-\lambda z_{1}$ in Eq. (2.24) to eliminate $P^{*}\left(\theta, z_{1}, z_{2}\right)$ and get

$$
\begin{aligned}
{\left[z_{1}-\right.} & \left.\left(1-p+p z_{2}\right) S^{*}\left(\lambda-\lambda z_{1}\right)\right] P\left(0, z_{1}, z_{2}\right) \\
= & \lambda z_{1}\left(z_{1}-1\right) S^{*}\left(\lambda-\lambda z_{1}\right) U\left(z_{2}\right) \\
& +p S^{*}\left(\lambda-\lambda z_{1}\right)\left[z_{1}^{T} D^{*}\left(\lambda-\lambda z_{1}\right)-z_{2}^{T}\right] P_{\cdot, T-1}\left(0, z_{1}\right) .
\end{aligned}
$$


Use $z_{2}=\Phi\left(z_{1}\right)=\left(z_{1}-(1-p) S^{*}\left(\lambda-\lambda z_{1}\right)\right) /\left(p S^{*}\left(\lambda-\lambda z_{1}\right)\right)$ in Eq. (2.26) to eliminate $P\left(0, z_{1}, z_{2}\right)$ and get

$$
\begin{gathered}
P_{\cdot, T-1}\left(0, z_{1}\right)=\frac{\lambda z_{1}\left(1-z_{1}\right) U\left[\Phi\left(z_{1}\right)\right]}{p\left[z_{1}^{T} D^{*}\left(\lambda-\lambda z_{1}\right)-\Phi^{T}\left(z_{1}\right)\right]} . \\
\Phi(1)=\left.\Phi\left(z_{1}\right)\right|_{z_{1}=1}=\left.\frac{z_{1}-(1-p) S^{*}\left(\lambda-\lambda z_{1}\right)}{p S^{*}\left(\lambda-\lambda z_{1}\right)}\right|_{z_{1}=1}=1 .
\end{gathered}
$$

We reserve the following quantities for later use:

$$
\begin{gathered}
\Phi^{(1)}(1)=\left.\frac{\mathrm{d}}{\mathrm{d} z_{1}} \Phi\left(z_{1}\right)\right|_{z_{1}=1}=\frac{1-\rho}{p} \quad(\rho=\lambda E(S)), \\
\Phi^{(2)}(1)=\left.\frac{\mathrm{d}^{2}}{\mathrm{~d} z_{1}^{2}} \Phi\left(z_{1}\right)\right|_{z_{1}=1}=-\frac{2 \rho(1-\rho)+\lambda^{2} E\left(S^{2}\right)}{p} .
\end{gathered}
$$

Using Eq. (2.27) in (2.26), we obtain

$$
\begin{aligned}
& P\left(0, z_{1}, z_{2}\right) \\
& =\lambda z_{1}\left(1-z_{1}\right) S^{*}\left(\lambda-\lambda z_{1}\right) \\
& \quad \times \frac{\left\{\left[\Phi^{T}\left(z_{1}\right)-z_{1}^{T} D^{*}\left(\lambda-\lambda z_{1}\right)\right] U\left(z_{2}\right)+\left[z_{1}^{T} D^{*}\left(\lambda-\lambda z_{1}\right)-z_{2}^{T}\right] U\left[\Phi\left(z_{1}\right)\right]\right\}}{\left[z_{1}-\left(1-p+p z_{2}\right) S^{*}\left(\lambda-\lambda z_{1}\right)\right]\left[z_{1}^{T} D^{*}\left(\lambda-\lambda z_{1}\right)-\Phi^{T}\left(z_{1}\right)\right]} .
\end{aligned}
$$

Using Eqs. (2.27) and (2.31) in (2.24) and (2.25), we get

$$
\begin{aligned}
& P^{*}\left(\theta, z_{1}, z_{2}\right) \\
& =\lambda z_{1}\left(1-z_{1}\right)\left[S^{*}(\theta)-S^{*}\left(\lambda-\lambda z_{1}\right)\right] \\
& \times\left\{\frac{U\left(z_{2}\right)\left[z_{1}^{T} D^{*}\left(\lambda-\lambda z_{1}\right)-\Phi^{T}\left(z_{1}\right)\right]+U\left[\Phi\left(z_{1}\right)\right]\left[z_{2}^{T}-z_{1}^{T} D^{*}\left(\lambda-\lambda z_{1}\right)\right]}{\left(\theta-\lambda+\lambda z_{1}\right)\left[z_{1}-\left(1-p+p z_{2}\right) S^{*}\left(\lambda-\lambda z_{1}\right)\right]\left[z_{1}^{T} D^{*}\left(\lambda-\lambda z_{1}\right)-\Phi^{T}\left(z_{1}\right)\right]}\right\}
\end{aligned}
$$


and

$$
Q^{*}\left(\theta, z_{1}\right)=\frac{\lambda\left(1-z_{1}\right) U\left[\Phi\left(z_{1}\right)\right]\left[D^{*}(\theta)-D^{*}\left(\lambda-\lambda z_{1}\right)\right]}{\left(\theta-\lambda+\lambda z_{1}\right)\left[\Phi^{T}\left(z_{1}\right)-z_{1}^{T} D^{*}\left(\lambda-\lambda z_{1}\right)\right]}
$$

Note that $P^{*}\left(\theta, z_{1}, z_{2}\right)$ is the triple joint transform of the number of parts in the main buffer, the number of parts in the feedback buffer, the remaining processing time and the probability that the operator is processing. $Q^{*}\left(\theta, z_{1}\right)$ is the joint transform of the number of parts in the main buffer, the remaining dispatching time and the probability that the operator is dispatching. We define $P\left(z_{1}, z_{2}\right)$ as follows:

$$
P\left(z_{1}, z_{2}\right)=U\left(z_{2}\right)+P^{*}\left(0, z_{1}, z_{2}\right)+z_{2}^{T} Q^{*}\left(0, z_{1}\right) .
$$

Then, we get

$$
\begin{aligned}
P\left(z_{1}, z_{2}\right) & \\
= & U\left(z_{2}\right)+P^{*}\left(0, z_{1}, z_{2}\right)+z_{2}^{T} Q^{*}\left(0, z_{1}\right) \\
= & \frac{U\left(z_{2}\right) S^{*}\left(\lambda-\lambda z_{1}\right)\left[z_{1}^{T} D^{*}\left(\lambda-\lambda z_{1}\right)-\Phi^{T}\left(z_{1}\right)\right]\left[\left(z_{1}-1\right)-p\left(z_{2}-1\right)\right]}{\left[z_{1}-\left(1-p+p z_{2}\right) S^{*}\left(\lambda-\lambda z_{1}\right)\right]\left[z_{1}^{T} D^{*}\left(\lambda-\lambda z_{1}\right)-\Phi^{T}\left(z_{1}\right)\right]} \\
& +\frac{U\left[\Phi\left(z_{1}\right)\right] z_{2}^{T}\left[\left(z_{1}-1\right)-p\left(z_{2}-1\right)\right] S^{*}\left(\lambda-\lambda z_{1}\right)}{\left[z_{1}-\left(1-p+p z_{2}\right) S^{*}\left(\lambda-\lambda z_{1}\right)\right]\left[z_{1}^{T} D^{*}\left(\lambda-\lambda z_{1}\right)-\Phi^{T}\left(z_{1}\right)\right]} \\
& +\frac{U\left[\Phi\left(z_{1}\right)\right] z_{1}\left(z_{1}^{T}-z_{2}^{T}\right) D^{*}\left(\lambda-\lambda z_{1}\right)}{\left[z_{1}-\left(1-p+p z_{2}\right) S^{*}\left(\lambda-\lambda z_{1}\right)\right]\left[z_{1}^{T} D^{*}\left(\lambda-\lambda z_{1}\right)-\Phi^{T}\left(z_{1}\right)\right]} \\
& +\frac{U\left[\Phi\left(z_{1}\right)\right]\left[z_{2}^{T}\left(1-p+p z_{2}\right)-z_{1}^{T+1}\right] S^{*}\left(\lambda-\lambda z_{1}\right) D^{*}\left(\lambda-\lambda z_{1}\right)}{\left[z_{1}-\left(1-p+p z_{2}\right) S^{*}\left(\lambda-\lambda z_{1}\right)\right]\left[z_{1}^{T} D^{*}\left(\lambda-\lambda z_{1}\right)-\Phi^{T}\left(z_{1}\right)\right]}
\end{aligned}
$$

We note that $z_{2}$ in $P\left(z_{1}, z_{2}\right)$ represents not only the number of parts in the feedback buffer but also the parts being dispatched.

\section{PeRFormance meAsuRes}

In this section, we develop various performance measures.

\subsection{State Probabilities}

Let $\theta=0$ and $z_{1}=z_{2}=1$ in Eq. (2.32) to obtain the probability that the operator is processing 


$$
P_{\text {proc }}=P^{*}(0,1,1)=\frac{\rho \cdot T \cdot U(1)}{[(1-p-\rho) T-\lambda p E(D)]} .
$$

Let $\theta=0$ and $z_{1}=1$ in Eq. (2.33) to obtain the probability that the operator is dispatching

$$
P_{\mathrm{disp}}=Q^{*}(0,1)=\frac{\lambda p E(D) U(1)}{[(1-p-\rho) T-\lambda p E(D)]} .
$$

From $P_{\text {idle }}+P_{\text {proc }}+P_{\text {disp }}=1$, we get

$$
P_{\text {idle }}=U(1)=\frac{(1-p-\rho) T-\lambda p E(D)}{(1-p) T}=1-\left(\frac{\rho T+\lambda p E(D)}{(1-p) T}\right) .
$$

Thus, we have

$$
\begin{gathered}
P_{\text {proc }}=\frac{\rho}{1-p}, \\
P_{\text {disp }}=\frac{\lambda p E(D)}{(1-p) T} .
\end{gathered}
$$

From Eq. (3.1), the stability condition becomes

$$
\frac{\rho T+\lambda p E(D)}{(1-p) T}<1
$$

\subsection{Mean Number of Parts}

The steady-state mean number of parts in the main buffer becomes

$$
\begin{aligned}
L_{\mathrm{M}}=E & \left(N_{\mathrm{M}}\right)=\left.\frac{\mathrm{d}}{\mathrm{d} z_{1}} P\left(z_{1}, 1\right)\right|_{z_{1}=1} \\
=\{ & \lambda^{2}(1-\rho)(1-p) T^{2} E\left(S^{2}\right) \\
& +\lambda^{2} p E\left(D^{2}\right)[(1-\rho) T+p(1-\lambda) E(D)+p T \cdot(E(S)-1)] \\
& +(1-\rho) T\left[\lambda E(D)\left((1-T)\left(1-p^{2}-\rho\right)+2 \lambda p^{2} E(D)+2 p \rho T\right)\right. \\
& +\rho T \cdot((3-T)(1-p)+\rho(2 p+T-3))] \\
& \left.+2(1-p)(1-\rho) T \cdot[\lambda E(D)+\rho T] U^{(1)}(1)\right\} \\
& /\{2(1-p)(1-\rho) T \cdot[(1-p-\rho) T-\lambda p E(D)]\} .
\end{aligned}
$$


The steady-state mean number of parts being dispatched is simply

$$
L_{\mathrm{D}}=T \cdot P_{\text {disp }}=\frac{\lambda p E(D)}{(1-p)} .
$$

We notice the following identity:

$$
\begin{aligned}
L_{\mathrm{F}}+L_{\mathrm{D}} & =E\left(N_{\mathrm{F}}+N_{\mathrm{D}}\right)=\left.\frac{\mathrm{d}}{\mathrm{d} z_{2}} P\left(1, z_{2}\right)\right|_{z_{2}=1} \\
& =U^{(1)}(1)+\frac{2 \lambda p E(D)+(T-1) \rho}{2(1-p)} .
\end{aligned}
$$

Thus, we get the mean number of parts in the feedback buffer,

$$
L_{\mathrm{F}}=U^{(1)}(1)+\frac{(T-1) \rho}{2(1-p)} .
$$

Computation of $U^{(1)}(1)$ in Eqs. (3.5), (3.7) and (3.8) can be found in the Appendix.

\subsection{Mean System Sojourn Time}

Steady-state mean total number of parts in the system becomes

$$
\begin{aligned}
& L=L_{\mathrm{M}}+L_{\mathrm{F}}+L_{\mathrm{D}} \\
& =\frac{(1-p-\rho) T\left[2 \rho T \cdot(1-\rho)+\lambda^{2} p E\left(D^{2}\right)+\lambda^{2} T \cdot E\left(S^{2}\right)\right]}{2(1-p-\rho)(1-p) U(1) T^{2}} \\
& +\frac{\lambda\left[\lambda p^{2}(1-\lambda) E\left(D^{2}\right)+(1-p-\rho)(1+p-\rho) T-(1-p-\rho)^{2} T^{2}\right] E(D)}{2(1-p-\rho)(1-p) U(1) T^{2}} \\
& +\frac{2(1-p) T \cdot[(1-p) T \cdot U(1)+\lambda(1-\rho) E(D)] U^{(1)}(1)}{2(1-p-\rho)(1-p) U(1) T^{2}}
\end{aligned}
$$

From Little's law, the mean system sojourn time becomes

$$
\begin{aligned}
& W=\frac{L}{\lambda} \\
& =\frac{(1-p-\rho) T\left[2 \rho T \cdot(1-\rho)+\lambda^{2} p E\left(D^{2}\right)+\lambda^{2} T \cdot E\left(S^{2}\right)\right]}{2 \lambda(1-p-\rho)(1-p) U(1) T^{2}} \\
& +\frac{\lambda\left[\lambda p^{2}(1-\lambda) E\left(D^{2}\right)+(1-p-\rho)(1+p-\rho) T-(1-p-\rho)^{2} T^{2}\right] E(D)}{2 \lambda(1-p-\rho)(1-p) U(1) T^{2}} \\
& +\frac{2(1-p) T \cdot[(1-p) T \cdot U(1)+\lambda(1-\rho) E(D)] U^{(1)}(1)}{2 \lambda(1-p-\rho)(1-p) U(1) T^{2}}
\end{aligned}
$$


TABLE I Comparison of our analytical results and the simulation estimates $(\rho=0.8, p=0.01, T=5)$. Processing time: $s(x)=\mu \mathrm{e}^{-\mu x}, E(S)=$ $1 / \mu=1.0$. Dispatching time: $d(x)=\beta \mathrm{e}^{-\beta x}, E(D)=1 / \beta=0.1$

\begin{tabular}{lclc}
\hline & Simulation & Our results & Relative error $(\%)$ \\
\hline$L_{\mathrm{M}}$ & 4.27429 & 4.28944 & 0.35319 \\
$L_{\mathrm{F}}$ & 2.02372 & 2.01712 & -0.32720 \\
$L_{\mathrm{D}}$ & -0.00080 & 0.00081 & 1.23457 \\
$W$ & 7.88093 & 7.88419 & 0.04135 \\
Dispatching rate & 0.00160 & 0.00162 & 1.23457 \\
$U_{0}$ & 0.0329918 & 0.0323645 & -1.93824 \\
$U_{1}$ & 0.0390427 & 0.0387689 & -0.70624 \\
$U_{2}$ & 0.0399761 & 0.0400044 & 0.07074 \\
$U_{3}$ & 0.0401166 & 0.0403028 & 0.46200 \\
$U_{4}$ & 0.0407969 & 0.0403175 & -1.18906 \\
\hline
\end{tabular}

\subsection{Dispatching Rate}

A dispatching occurs as soon as a finished part is fed back when there are $T-1$ parts in the feedback buffer. Thus the dispatching rate, $\alpha(T)$, which is defined as the mean number of dispatching per unit time, can be obtained as

$$
\alpha(T)=p P_{\cdot, T-1}(0,1)=\frac{\lambda p}{T(1-p)},
$$

or, it can be obtained from $P_{\text {disp }} / E(D)$.

\subsection{Model Verification}

We compare our results with simulation estimates. Table I shows a comparison between our analytical performance measures and the simulation estimates. In almost all of our extensive computer experiments, the relative errors were within $1 \%$.

\section{EFFECTS OF THE DISPATCHING TIME}

In this section, we evaluate the effects of the dispatching time on the system performance. 


\subsection{Effects on the Server Utilization and Dispatching Rate}

First, it is easily seen that the operator utilization $\rho_{\mathrm{D}}=P_{\text {proc }}+P_{\text {disp }}$ is greater than $\rho_{\mathrm{O}}$, the utilization under zero dispatching time $(E(D)=0)$. It tells us that if we do not incorporate the dispatching time into the modeling process, it is likely that the operator utilization is underestimated by the amount

$$
\rho_{\mathrm{D}}-\rho_{\mathrm{O}}=P_{\mathrm{disp}}=E(D) \cdot \alpha(T)=\frac{\lambda p E(D)}{(1-p) T} .
$$

The existence of the dispatching time does not alter the dispatching rate. This is because the operator has to process $\lambda$ parts per unit time in the long run, regardless of the dispatching time.

\subsection{Effects of the Mean and Variance of the Dispatching Time}

Figures 2 and 3 show the change of the mean number of parts in each buffer as mean dispatching time varies for two different values of $\rho=\lambda E(S)$. It is seen that $E(D)$ increases $L_{\mathrm{M}}$, which is obvious from the fact that longer dispatching time increases the number of new parts that enter the main buffer and wait. $E(D)$ also increases $L_{\mathrm{D}}$. This is due to the fact that larger $E(D)$ increases the time proportion taken by the dispatching time.

Figures 4 and 5 show the change of the mean number of parts in each buffer as the variance of the dispatching time changes with fixed

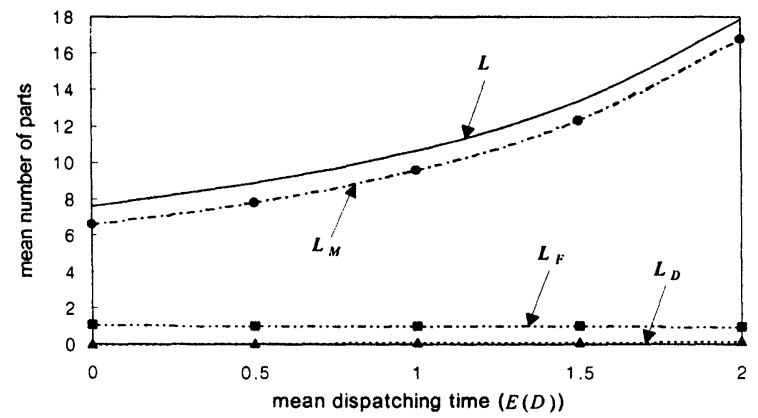

FIGURE 2 Mean number of parts as a function of $E(D)(\rho=0.8, p=0.1, T=3)$. Processing time: $s(x)=\mu^{2} x \mathrm{e}^{-\mu x}, E(S)=(2.0 / \mu)=1.0$. Dispatching time: $d(x)=\beta \mathrm{e}^{-\beta x}$, $E(D)=1 / \beta$. 


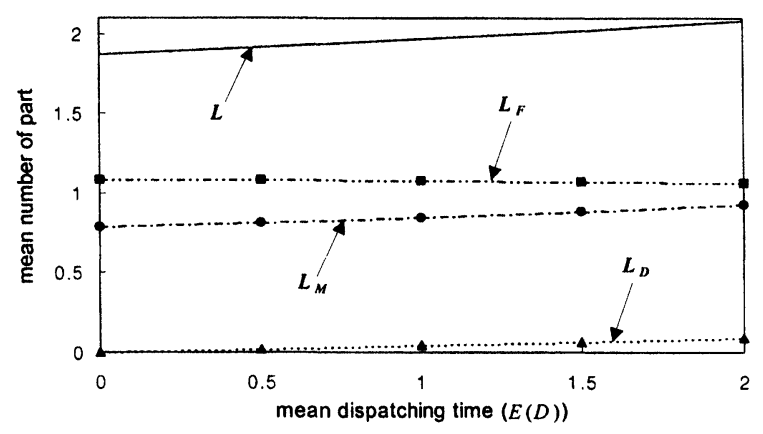

FIGURE 3 Mean number of parts as a function of $E(D)(\rho=0.4, p=0.1, T=3)$. Processing time: $s(x)=\mu^{2} x \mathrm{e}^{-\mu x}, E(S)=(2.0 / \mu)=1.0$. Dispatching time: $d(x)=\beta \mathrm{e}^{-\beta x}$, $E(D)=1 / \beta$.

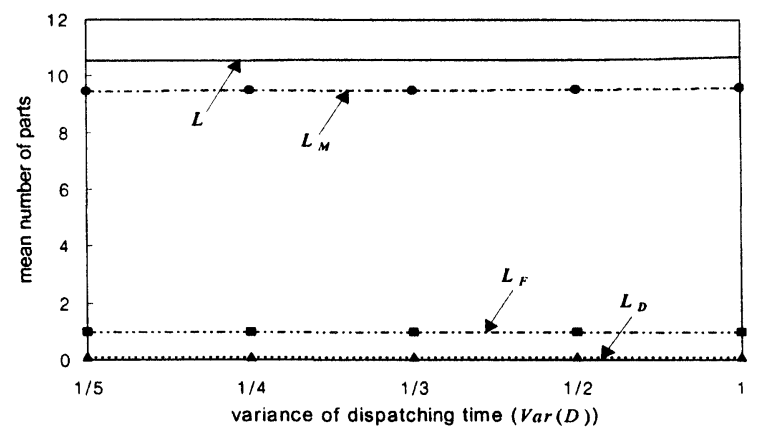

FIGURE 4 Mean number of parts as a function of $\operatorname{Var}(D)(\rho=0.8, p=0.1, T=3)$. Processing time: $s(x)=\mu^{2} x \mathrm{e}^{-\mu x}, \quad E(S)=(2.0 / \mu)=1.0$. Dispatching time: $d(x)=$ $\left(\beta^{k} x^{k-1} \mathrm{e}^{-\beta x}\right) /(k-1) !, E(D)=(k / \beta)=1.0$.

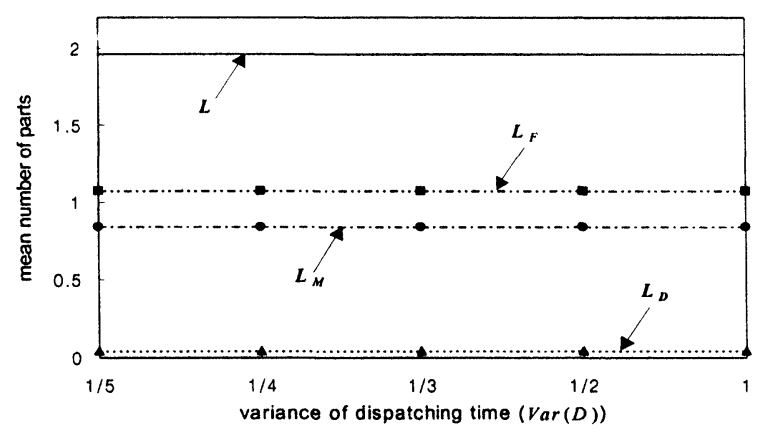

FIGURE 5 Mean number of parts as a function of $\operatorname{Var}(D)(\rho=0.4, p=0.1, T=3)$. Processing time: $s(x)=\mu^{2} x \mathrm{e}^{-\mu x}, \quad E(S)=(2.0 / \mu)=1.0$. Dispatching time: $d(x)=$ $\left(\beta^{k} x^{k-1} \mathrm{e}^{-\beta x}\right) /(k-1) !, E(D)=(k / \beta)=1.0$. 
mean dispatching time. For all cases, the variance is not significant. This may give us a guideline in controlling the dispatching time. The operator, to reduce the work-in-process inventory, has to reduce the mean dispatching time regardless of the variability of the dispatching time.

\section{DETERMINING THE OPTIMAL SIZE OF THE FEEDBACK BUFFER}

In this section, we determine $T^{*}$, the optimal size of the feedback buffer under a cost structure. Let us consider the following costs:

$C_{\mathrm{w}}$ : cost of holding a part in the system for a unit time, $C_{\mathrm{D}}$ : dispatching cost.

The overall average cost per unit time becomes

$$
\begin{aligned}
& A C(T)=C_{\mathrm{W}} \cdot L+C_{\mathrm{D}} \cdot \alpha(T) \\
& =C_{\mathrm{W}} \cdot\left\{\frac{(1-p-\rho) T\left[2 \rho T \cdot(1-\rho)+\lambda^{2} p E\left(D^{2}\right)+\lambda^{2} T \cdot E\left(S^{2}\right)\right]}{2(1-p-\rho)(1-p) U(1) T^{2}}\right. \\
& +\frac{\lambda\left[\lambda p^{2}(1-\lambda) E\left(D^{2}\right)+(1-p-\rho)(1+p-\rho) T-(1-p-\rho)^{2} T^{2}\right] E(D)}{2(1-p-\rho)(1-p) U(1) T^{2}} \\
& \left.+\frac{2(1-p) T \cdot[(1-p) T \cdot U(1)+\lambda(1-\rho) E(D)] U^{(1)}(1)}{2(1-p-\rho)(1-p) U(1) T^{2}}\right\} \\
& +C_{\mathrm{D}} \cdot \frac{\lambda p}{T(1-p)} .
\end{aligned}
$$

$U^{(1)}(1)$ in the above equation is dependent on $T$ (calculation of $U^{(1)}(1)$ can be found in the Appendix). Thus it is extremely difficult to see whether $A C(T)$ is convex or unimodal. But our comprehensive computer experiments convinced us that it is convex and unimodal.

Figure 6 shows the value of $A C(T)$ as $T$ varies for a particular set of parameters. In this example, $T^{*}=4$ is the optimal size of the feedback buffer. 


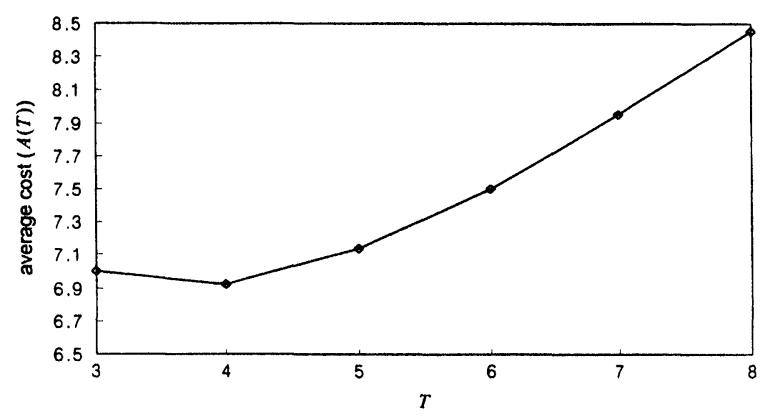

FIGURE 6 Average cost of $T$ varies $\left(\rho=0.7, p=0.1, C_{\mathrm{W}}=1, C_{\mathrm{D}}=100\right)$. Processing time: $s(x)=\mu^{2} x \mathrm{e}^{-\mu x}, E(S)=(2.0 / \mu)=1.0$; Dispatching time: $d(x)=\beta \mathrm{e}^{-\beta x}, E(D)=1 / \beta$.

\section{RESEARCH SUMMARY}

In this research, we analyzed a production system with an intermediate feedback buffer and positive dispatching time. Various performance measures were derived using transform methods. We found the optimal buffer size that minimizes the overall average operating cost.

The effects of the dispatching time on performance measures were discussed. It was that variance of the dispatching time does not play a significant role in the operational behavior of the system. Thus, if one wants to reduce the work-in-process inventory, he has to reduce the mean dispatching time rather than paying attention to the variability of the dispatching time. It was also found that dispatching time must be incorporated into the modeling of the system. Otherwise, work-inprocess inventory would be severely underestimated.

\section{Acknowledgment}

This research was supported by Non-directed Research Fund, Korea Research Foundation.

\section{References}

[1] G.R. D'avignon and R.L. Disney, "Queues with instantaneous feedback”, Management Science, 1977, 24(2), 168-180.

[2] R.L. Disney, "A note on sojourn times in $M / G / 1$ queues with instantaneous Bernoulli feedback”, Naval Research Logistics Quarterly, 1981, 28(4), 679-684. 
[3] R.L. Disney, D.C. McNickle and B. Simon, "The $M / G / 1$ queue with instantaneous Bernoulli feedback", Naval Research Logistics Quarterly, 1980, 27, 635-644.

[4] R.D. Foley and R.L. Disney, "Queue with delayed feedback", Advances in Applied Probability, 1983, 15, 162-182.

[5] H.W. Lee and D.W. Seo, "Design of a production system with feedback buffer", QUESTA, 1997, 26(1), 187-202.

[6] B. Pourbabai, "An $M / G / 1 / K$ queueing system with delayed Bernoulli feedback", Personal Correspondence, 1987.

[7] L.E. Schrage, "The queue $M / G / 1$ with feedback to lower priority queues", Management Science, 1967, 13(7), 466-474.

[8] L. Takács, "A single server queue with feedback”, Bell Syst. Tech. J., 1963, 42, 505-519.

[9] T. Takine, H. Takagi and T. Hasegawa, "Sojourn times in vacation and polling systems with Bernoulli feedback", J. Appl. Prob., 1991, 28, 422-432.

[10] M.A. Wortman, R.L. Disney and P.C. Kiessler, "The $M / G / 1$ Bernoulli feedback queue with vacations", Queueing Systems, 1991, 9, 353-364.

\section{APPENDIX}

Computing $U_{k}(k=0,1, \ldots, T-1)$

Performance measures contain $U^{(1)}(1)$ which is an unknown quantity. $U^{(1)}(1)$ is determined once $\left\{U_{0}, U_{1}, \ldots, U_{T-1}\right\}$ are found. We apply the well-known Rouche's theorem for this purpose.

We use Eq. (2.34) to obtain $\left\{U_{0}, U_{1}, \ldots, U_{T-1}\right\} . z_{1}-S^{*}\left(\lambda-\lambda z_{1}\right)$ has a zero $z_{1}=1$. Also, one of the zeros of $z_{1}^{T} D^{*}\left(\lambda-\lambda z_{1}\right)-\Phi^{T}\left(z_{1}\right)$ is $z_{1}=1$. Thus, we consider only $z_{1}^{T} D^{*}\left(\lambda-\lambda z_{1}\right)-\Phi^{T}\left(z_{1}\right)$. Let us define

$$
C_{1}(z)=S^{*}(\lambda-\lambda z)(z-1)\left[z^{T} D^{*}(\lambda-\lambda z)-\Phi^{T}(z)\right]
$$

and

$$
\begin{aligned}
C_{2}(z)= & (z-1) S^{*}(\lambda-\lambda z)+\left(z^{T+1}-z\right) D^{*}(\lambda-\lambda z) \\
& +\left(1-z^{T+1}\right) S^{*}(\lambda-\lambda z) D^{*}(\lambda-\lambda z) .
\end{aligned}
$$

From $U(z)=\sum_{j=0}^{T-1} U_{j} z^{j}=U_{0} z^{0}+U_{1} z^{1}+\cdots+U_{T-1} z^{T-1}$, we get

$$
U(1)=U_{0}+U_{1}+\cdots+U_{T-1} .
$$

Let the $T$ zeros of $z^{T} D^{*}(\lambda-\lambda z)-\Phi^{T}(z)$ on $|z| \leq 1 \quad$ be $z_{(1)}, z_{(2)}, \ldots, z_{(T-1)}$ and $z_{(0)}=1$. The numerator of Eq. (2.34) evaluated 
at these zeros must vanish. Thus we can set up $T$ simultaneous equations as follows:

$$
\begin{gathered}
U(1)=\frac{[(1-p-\rho) T-\lambda p E(D)]}{(1-p) T}, \\
C_{1}\left(z_{(j)}\right) U(1)+C_{2}\left(z_{(j)}\right) U\left[\Phi\left(z_{(j)}\right)\right]=0, \quad j=1,2, \ldots, T-1 .
\end{gathered}
$$

Arranging terms yield

$$
\begin{gathered}
U_{0}+U_{1}+\cdots+U_{T-1}=\frac{[(1-p-\rho) T-\lambda p E(D)]}{(1-p) T} \\
{\left[C_{1}\left(z_{(j)}\right)+C_{2}\left(z_{(j)}\right)\right] U_{0}+\left[C_{1}\left(z_{(j)}\right)+C_{2}\left(z_{(j)}\right) \Phi\left(z_{(j)}\right)\right] U_{1}} \\
+\left[C_{1}\left(z_{(j)}\right)+C_{2}\left(z_{(j)}\right) \Phi\left(z_{(j)}\right)^{2}\right] U_{2} \\
+\cdots+\left[C_{1}\left(z_{(j)}\right)+C_{2}\left(z_{(j)}\right) \Phi\left(z_{(j)}\right)^{T-1}\right] U_{T-1}=0 \\
(j=1,2, \ldots, T-1) .
\end{gathered}
$$

We can obtain $\left\{U_{0}, U_{1}, \ldots, U_{T-1}\right\}$ by solving the above equations. Then $U^{(1)}(1)$ is completely determined. 


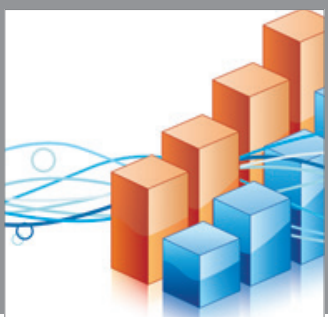

Advances in

Operations Research

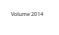

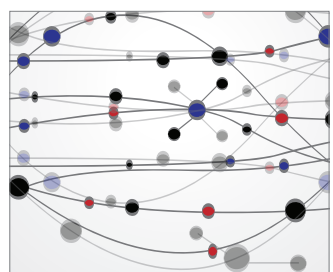

\section{The Scientific} World Journal
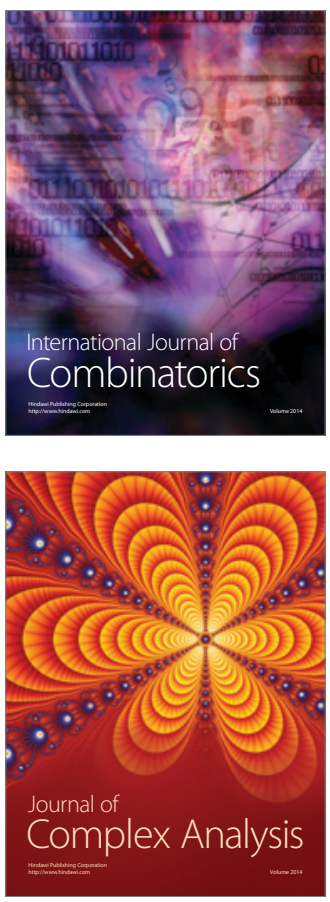

International Journal of

Mathematics and

Mathematical

Sciences
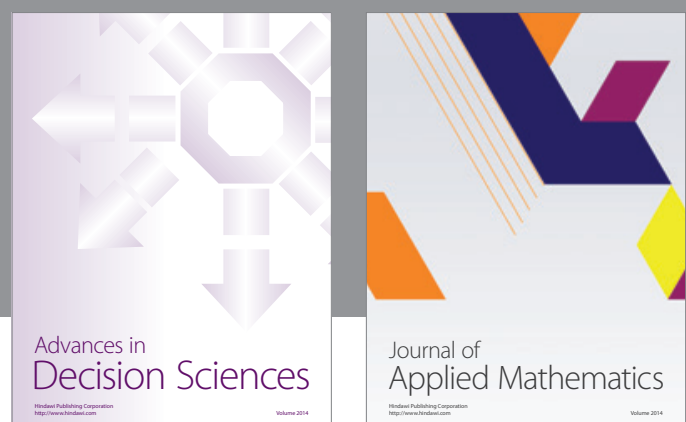

Journal of

Applied Mathematics
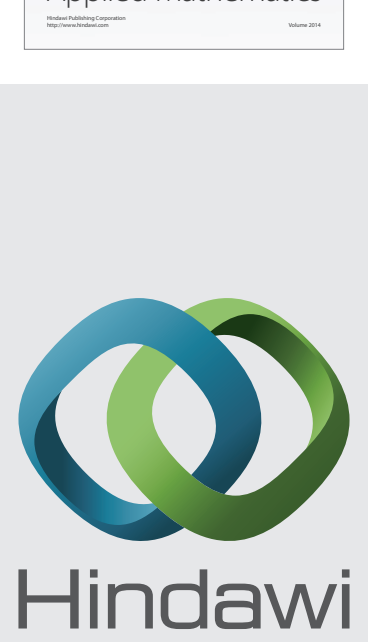

Submit your manuscripts at http://www.hindawi.com
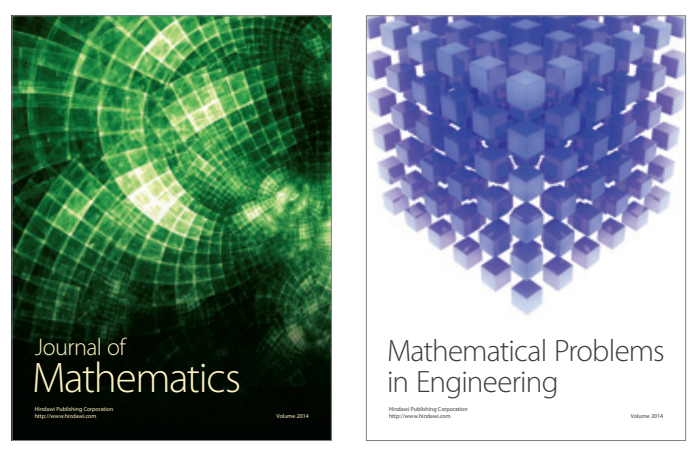

Mathematical Problems in Engineering
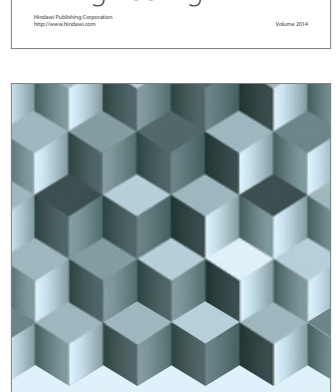

Journal of

Function Spaces
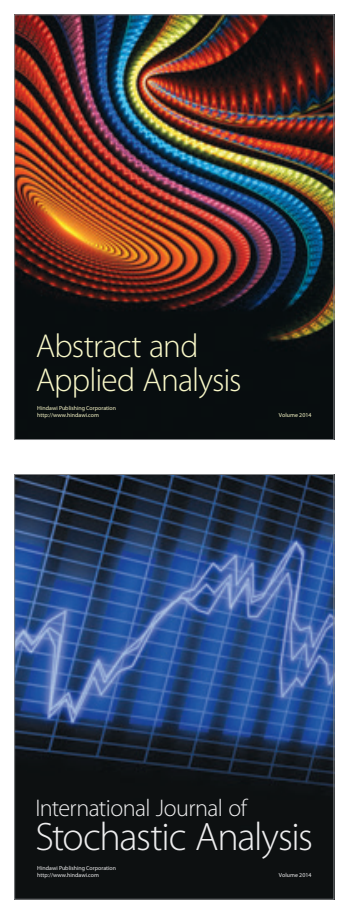

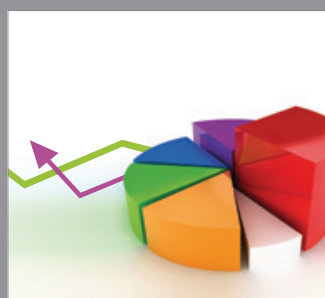

ournal of

Probability and Statistics

Promensencen
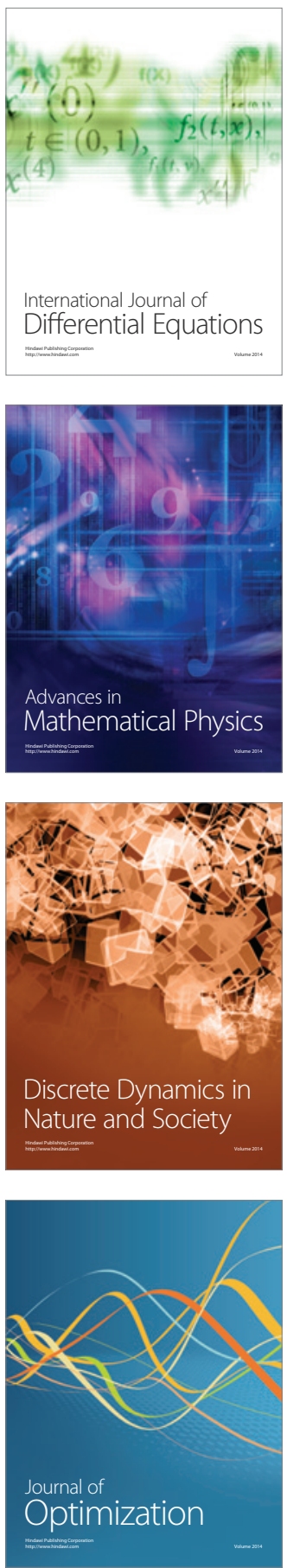